\title{
A Side-Constrained Peer-to-Peer Carpooling Stochastic User Equilibrium Model
}

\author{
Ruimin MA, Lifei YAO
}

\begin{abstract}
Peer-to-peer (P2P) carpooling has become an effective way to utilize the idle car capacity and ease traffic congestion. However, the interactive relationship between carpooling and traffic congestion has not been fully quantified. To make up for this gap, this paper constructs a side-constrained P2P carpooling stochastic user equilibrium model to disclose the effects of carpooling on traffic congestion. Next, the proposed model was transformed into a linear constrained minimization problem, and the problem was proved to have a unique solution. The case study shows that the travellers prefer carpooling at a high fuel price and a low inconvenient cost.
\end{abstract}

Keywords: linear constrained minimization; peer-to-peer (P2P) carpooling; side-constrained; stochastic user equilibrium; traffic congestion

\section{INTRODUCTION}

The population boom, coupled with urban sprawl, has intensified the traffic congestion in big cities across the globe. In its 2012 Urban Mobility Report, the Texas A \& M Transportation Institute pointed out that each American commuter faced a delay of $38 \mathrm{~h}$ and a congestion cost of $820 \$$ in 2012 , putting the total congestion cost in the US at 120 billion USD [1]. In 2015, the congestion cost in Beijing, China amounted to $115 \mathrm{USD} /$ month, i.e. RMB 1385 USD/year, per commuter. Against this backdrop, governments around the world have been paying efforts to ease traffic congestion, but the effect is poor due to the growing number and low occupancy of private cars. In Europe, the car occupancy is only 1,13 people per car on the way to work or other places (EEA, 2010, European Environment Agency. Occupancy rates of passenger vehicles. Technical report. Retrieved from European Environment Agency). In the US, the private cars are less occupied, as only $10 \%$ Americans choose carpooling to work (US-DOT, 2011, U.S. DOT / Federal Highway Administration (FHWA). Our Nation's Highways). If the unoccupied transport ability of private cars could be utilized, the traffic congestion would be effectively alleviated. This provides a good opportunity to peer-topeer (P2P) carpooling: the sharing of car journeys so that more than one person travels in a car, and prevents the need for others to have to drive to a location themselves. In fact, P2P carpooling has become increasingly popular thanks to the proliferation of sharing economy and the maturation of positioning, mobile network and payment technologies.

The existing studies have shown that P2P carpooling can enhance the occupancy of private cars, and reduce total mileage, environmental pollution and exhaust emissions. However, most of them have only statistically quantified how much P2P carpooling eases traffic congestion, failing to identify the relationship between the two factors. Understanding this relationship is critical to the evaluation of carpooling providers, and the formulation of proper management and incentive measures. Under the background of sharing economy, this paper attempts to disclose the relationship between P2P carpooling and traffic congestion.

\section{LITERATURE REVIEW}

Ridesharing has long been a research hotspot in the transport field, previous studies have discussed the ridematching algorithms for ridesharing systems [2-3], dynamic ridesharing pricing [4-5], labour supply [6], trust among peers [7-8], socio-economic impacts of ridesharing services [9], environmental effects of ridesharing [10-11]

Some scholars began to pay close attention to the impact of ridesharing on traffic congestion. There are different voices on distinguishing an impact of ridesharing on traffic. Boosters claim that the service might help to mitigate traffic congestion by providing travellers with a more efficient transportation mode and by reducing car use as well as car ownership. Based on mobile data, Alexander and González [12] discovered that ridesharing can significantly shorten the duration of traffic congestion under a high matching rate. Rayle et. al [13] argued that ridesharing only supplements public transport, though being an alternative to long-distance transport. Li et. al suggested that Uber eased the traffic congestion in American cities by reducing the idle rate of parking lots and curbing the growth in car ownership [14-15]. Opponents on the other hand criticize that the service would worsen current traffic, introduce more vehicular trips, compete public transits, mislead consumers with varying price system and cater to only specific groups of people who are well-educated and young. Erhardt et al. [16] examined whether transportation network companies (such as Uber and Lyft) live up to their stated vision of reducing congestion in major cities, and found that transportation network companies are the biggest contributor to growing traffic congestion in San Francisco. Most of the above studies are statistical analysis on how ridesharing affects traffic congestion, failing to reveal the exact influence mechanism and the quantitative relation between ridesharing and traffic. The $\mathrm{P} 2 \mathrm{P}$ ridesharing considered in this paper is different from the traditional ridesharing (such as Uber), which allows private drivers to share the spare seats with other travellers for cost-saving purposes, who fundamentally differ from Taxi drivers who cruise around on the road for profit purposes.

Few studies have quantified the interactive relationship between $\mathrm{P} 2 \mathrm{P}$ carpooling and traffic congestion. Focusing on autostrada with or without highoccupancy vehicle (HOV) lanes, Yang and Huang [17] 
probed into the relationship between $\mathrm{P} 2 \mathrm{P}$ carpooling and the optimal congestion price, and compared the optimal and second-best pricing models; however, their research only applies to the case of single origin and single destination (SOSD) and allows each driver to take only one rider. Qian and Zhang [18] investigated the relationship between P2P carpooling and traffic congestion of multiple transport modes, namely, public transport, private car and carpooling, but their research is also limited to the SOSD transport network. Xu et al. [19] put forward a new traffic assignment model to find the relationship between P2P carpooling factors (e.g. price, the number of drivers and the number of riders) and traffic congestion in the case of multiple origins and multiple destinations (MOMD), assuming that the drivers and riders are assumed to have the same origin-destination (OD) pairs. In actual P2P carpooling, the drivers often take detours to pick up or drop off riders, indicating that the drivers and riders often have inconsistent OD pairs. In view of this shortage, $\mathrm{Xu}$ et al. [20] set up a traffic equilibrium model based on P2P carpooling, transformed the model into a hybrid complementary model, and applied the hybrid model to several cases with different network structures. The case study shows that more travellers prefer to act as carpooling drivers at a high price of $\mathrm{P} 2 \mathrm{P}$ carpooling. Nevertheless, the research of Xu et al. faces two problems: (1) the travel cost, an estimated value for carpooling participants, was taken as an exact value; (2) the link capacity was not considered and thus sometimes surpassed by the link flow.

\section{MATHEMATICAL MODEL}

In order to analyse the problem of P2P carpooling stochastic user equilibrium with sided-constrained, this paper makes the following assumptions: each traveller can act as a solo driver, a carpooling driver or a carpooling rider; the same traveller needs to pay different costs of the same arc on an OD pair for different transport modes; the rider alone cannot select between travel modes, but being supported by a carpooling driver.

\subsection{Network Description}

According to above assumptions, the author set up a super-network to distinguish between the travel modes, which can be regarded as the combination of the rider's network and the driver's network. The super-network is presented in Fig. 1, where $V^{\prime}$ is the set of riders' origins and destinations and $A^{\prime}$ is the set of origins and destinations shared by riders and drivers.

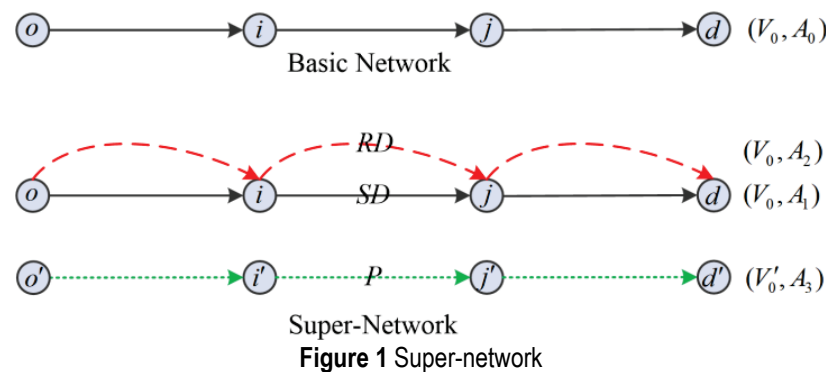

In Fig. $1, R D, S D$ and $P$ are respectively the abbreviation of carpooling driver, solo driver and passenger. For simplicity, it is also assumed that the role (i.e. driver or rider) of a traveller remains unchanged through the journey from the origin to the destination, and that each rider can only be served by one driver, i.e. the riders are reluctant to change cars. Under this assumption, any arc in the super-network is either driver or rider. As shown in Fig. 1, there are three types of arcs in the supernetwork, respectively denoted as $A_{1}, A_{2}$ and $A_{3}$. Under $A_{1}$, a solo driver continues to drive alone after passing through node $i$; Under $A_{2}$, a solo driver changes into a carpooling driver (i.e. picks up a rider) after passing through node $i$; Under $A_{3}$, a carpooling driver changes into a solo driver (i.e. drops off a rider).

\begin{tabular}{|c|c|}
\hline$G_{0}=\left(V_{0}, A_{0}\right)$ & $\begin{array}{l}\text { Basic network, where } V_{0} \text { is the set of nodes and } A_{0} \text { is } \\
\text { the set of arcs }\end{array}$ \\
\hline$G=(V, A)$ & $\begin{array}{l}\text { Super-network, where } V=V_{0} \cup V_{0}^{\prime} \text { is the set of nodes } \\
\text { and } A \text { is the set of arcs }\end{array}$ \\
\hline $\bar{O}, \bar{D} \subseteq V_{0}$ & $\begin{array}{c}\text { The set of origins and the set of destinations in the } \\
\text { basic network }\end{array}$ \\
\hline$O, D \subseteq V$ & $\begin{array}{l}\text { The set of origins and the set of destinations in the } \\
\text { super-network, } O=\bar{O} \cup O_{0}^{\prime} \text { and } D=\bar{D} \cup \bar{D}^{\prime}\end{array}$ \\
\hline$A_{1}, A_{2}, A_{3}$ & $\begin{array}{l}\text { The set of the arcs of solo drivers, the set of the arcs of } \\
\text { carpooling drivers, the set of arcs of riders; } \\
\qquad A=A_{1} \cup A_{2} \cup A_{3}\end{array}$ \\
\hline$a_{1}, a_{2}, a_{3}$ & The $\operatorname{arcs}$ in $A_{1}, A_{2}$ and $A_{3}, a_{j} \in A_{j}, j=1,2,3$ \\
\hline$K \subseteq O \times D$ & $\begin{array}{c}K=\left\{\left(o_{k}, d_{k}\right),\left(o^{\prime}{ }_{k}, d^{\prime}{ }_{k}\right) \mid o_{k} \in \bar{O}, d_{k} \in \bar{D}, o^{\prime} \in \overline{O^{\prime}}\right\} \\
\text { is the set of OD pairs }\end{array}$ \\
\hline$R_{k}$ & $\begin{array}{l}\text { The set of paths between OD pair } k \text {, i.e. the set of all } \\
\text { paths from } o_{k} \text {. to } d_{k} \text { or from } o_{k}^{\prime} \text { to } d^{\prime}{ }_{k}, r \in R_{k} \text { is one of } \\
\text { the paths }\end{array}$ \\
\hline
\end{tabular}

Let $G_{0}=\left(V_{0}, A_{0}\right)$ and $G=(V, A)$ be the basic network and the super-network, respectively. As shown in Fig. 1, $V$ is the set of nodes, $A$ is the set of arcs, $A_{1}$ is the set of the arcs of solo drivers (i.e. $(o, i),(i, j)$ and $(j, d)), A_{2}$ is the set of arcs of carpooling drivers $((o, i),(i, j)$ and $(j, d)), A_{3}$ is the set of arcs of riders (i.e. $(o, i),(i, j)$ and $(j, d))$. The details on the super-network are listed in Tab. 1.

Table 2 Parameters and variable

\begin{tabular}{|c|c|c|}
\hline \multirow{15}{*}{ Parameters } & $q_{k}$ & Demand of OD pair $k, k \in K$ \\
\hline & $u_{k}$ & Minimum expected travel cost of OD pair $k$ \\
\hline & $C$ & Maximum capacity of each driver \\
\hline & $t_{a}$ & Minimum time for $a \in A$ \\
\hline & $l_{a}$ & Length for $a \in A$ \\
\hline & $c_{a}$ & Traffic capacity for $a \in A$ \\
\hline & $x_{a}$ & Flow for $a \in A$ between OD pair $k \in K$ \\
\hline & $c_{a}^{k}$ & Travel cost for $a \in A$ between OD pair $k \in K$ \\
\hline & $y_{a}$ & Flow for $a \in A$ \\
\hline & $h_{k}^{r}$ & Flow of path $r$ between OD pair $k, r \in R_{k}$ \\
\hline & $c_{k}^{r}$ & $\begin{array}{c}\text { General travel cost of path } r \in R_{k} \text { between OD } \\
\text { pair } k\end{array}$ \\
\hline & $f_{a}$ & Cost for $a \in A$ \\
\hline & $y$ & Vector with components $y_{a}$ for $a \in A$ \\
\hline & $t t_{a}(y)$ & Driver's travel time cost for $a \in A_{1} \cup A_{2}$ \\
\hline & $t t_{a}^{\prime}(y)$ & Rider's travel time cost for $a \in A_{3}$ \\
\hline Variable & $\delta_{a r}$ & $\begin{array}{c}\text { If } \delta_{a r}=1, \text { then arc } a \text { lies on } r ; \text { if } \delta_{a r}=0, \text { then } \operatorname{arc} a \\
\text { does not lie on } r\end{array}$ \\
\hline
\end{tabular}

Since $A_{1}, A_{2} \subset V_{0} \times V_{0}$ and $A_{3} \subset V_{0}^{\prime} \times V_{0}^{\prime}$, any feasible path through the nodes in $V_{0} V_{0}$ can and only can go 
through the arcs in $A_{1} \cup A_{2}$ Similarly, any feasible path through the nodes in $V_{0}^{\prime}$ can and only can go through the $\operatorname{arcs}$ in $A_{3}$. Hence, no feasible path can go through the arcs in $A_{3}$ and $A_{1} \cup A_{2}$ at the same time. Tab. 2 lists the parameters and variable in this research.

\subsection{Cost Function}

The travel cost of a driver consists of three parts: travel time cost, fuel cost and inconvenient cost. On this basis, the cost function of carpooling can be expressed as follows.

\subsubsection{Congestion Cost of Drivers}

According to the classic Bureau of Public Roads (BPR) arc cost function [21], the congestion cost of drivers can be calculated based on the total number of drivers on the arc:

$t t_{a}(y)=t_{a}\left(1+\alpha\left(\frac{y_{a_{1}}+y_{a_{2}}}{c_{a}}\right)^{\beta}\right), a \in A_{1} \cup A_{2}$

where, $\alpha$ and $\beta$ are positive constants.

\subsubsection{Fuel Cost of Drivers}

The fuel cost takes up a huge part in the travel cost. It can be obtained by multiplying the arc length and the fuel price per unit length:

$M_{a}=\tau \cdot \rho \cdot l_{a}, a \in A_{0}$

where, $\rho$ is the fuel price per unit length; $\tau$ is the dimensional transformation parameter between cost and time. Both parameters are positive constants.

\subsubsection{Congestion Cost of Riders}

The congestion cost of riders can be computed by:

$t t_{a}^{\prime}(y)=t_{a}\left(1+\alpha\left(\frac{y_{a_{1}}+y_{a_{2}}+y_{a_{3}}}{c_{a}}\right)^{\beta}\right), a \in A_{3}$

where, the values of $\alpha$ and $\beta$ are the same as those in Eq. (1), assuming that the drivers and riders in P2P carpooling can tolerate the same length of congestion time.

\subsubsection{Payment of Riders}

The drivers joining the carpooling system aim to reduce the travel cost by charging fees to riders. Here, it is assumed that the drivers and riders go Dutch on the fuel cost of the shared arcs. The payment of carpooling riders can be calculated as:

$$
B_{a}^{p}(y)=\frac{1}{\gamma+1} M_{a}, a \in A_{3}
$$

where, the superscript $p$ denotes rider.

\subsubsection{Income of Drivers}

The driver's income equals the sum of the payments of all riders in his / her vehicle. Considering the match between drivers and riders, the number of riders per vehicle should be limited between 1 and the maximum capacity $C$ of the driver. Then, the income of drivers can be expressed as:

$$
B_{a}^{d}(y)=\gamma \cdot \frac{1}{\gamma+1} M_{a}, a \in A_{2}
$$

where, the superscript $d$ denotes driver; $\gamma \in(1, C)$ is the number of riders per vehicle.

\subsubsection{Inconvenient Cost of Drivers}

The inconvenient cost of drivers includes the extra travel time cost, the waiting time cost for picking up riders, and the cost to serve the riders. Thus, this cost can be formulated as:

$$
I_{a}^{d}(y)=\mu_{a}^{d} y_{a_{2}}+\pi_{a}^{d} y_{a_{3}}, a \in A_{2}
$$

\subsubsection{Inconvenient Cost of Riders}

Carpooling brings some inconveniences to the riders, such as the cost to travel from the origin to the pick-up point, the cost to wait for the driver, and the cost of detour to pick up or drop off other riders. Thus, this cost can be formulated as:

$$
I_{a}^{p}(y)=\mu_{a}^{p} y_{a_{2}}+\pi_{a}^{p} y_{a_{3}}, a \in A_{3}
$$

To sum up, the total travel cost of any traveller on arc $a \in A$ can be expressed as:

$$
f_{a}(y)=\left\{\begin{array}{l}
t t_{a}(y)+M_{a}(y), a \in A_{1} \\
t t_{a}(y)+M_{a}(y)-B_{a}^{d}(y)+I_{a}^{d}(y), a \in A_{2} \\
t t_{a}^{\prime}(y)+B_{a}^{p}(y)+I_{a}^{p}(y), a \in A_{3}
\end{array}\right.
$$

For any OD pairs, the cost $f_{a}(y)$ on the arc $a$ remains the same. If all the total travel costs on the arcs are independent, then the total path cost in the network can be obtained by adding up the cost of each arc:

$$
c_{k}^{r}(h)=\sum_{a \in A} \delta_{a r}^{k} f_{a}(y)
$$

where, $c_{k}^{r}(h)$ is the total cost of path $p ; \delta_{a r}^{k}$ is the relationship between arc $a$ and path $r$ (If $\delta_{a r}=1$, then arc $a$ lies on $r$; if $\delta_{a r}=0$, then $\operatorname{arc} a$ does not lie on $r$.); $h$ is the path flow corresponding to the arc flow $y$. 


\subsection{Side-Constrained P2P Carpooling Stochastic User Equilibrium Model}

To solve the inaccurate, random estimation of path cost of carpooling participants, this paper describes the path selection of these participants with the stochastic equilibrium model. Specifically, the Wardrop's user equilibrium principle was introduced into the P2P carpooling travel distribution, creating a side-constrained P2P carpooling stochastic user equilibrium model (SCP2P-CSUE model):

Arc flow constraints:

$y_{a}=\sum_{k \in K} x_{a}^{k}, \forall a \in A$

$y_{a} \leq c_{a}$

$x_{a}^{k} \geq 0, \forall a \in A, \forall k \in K$

Path flow constraints:

$x_{a}^{k}=\sum_{k \in K} \delta_{a r} h_{k}^{r}, \forall r \in R$

$h_{k}^{r} \geq 0$

OD demand constraints:

$\sum_{r \in R_{k}} h_{k}^{r}=q_{k}, k \in K$

$h_{k}^{r}=P_{k}^{r} q_{k}, k \in K$

where, $y_{a}=y_{a_{1}}+y_{a_{2}}+\gamma y_{a_{3}}$, with $\gamma \in(1,4)$ being the number of riders picked up or dropped off by a driver.

Eq. (11) shows the difference between our model and the traditional stochastic user equilibrium model. This equation was called by Patriksson and Larsson as the sideconstrained model and extended into the total Wardrop's equilibrium model named side-constrained traffic assignment model [22-23]. Nonetheless, the extended model only considers side-constrained determined user equilibrium and does not apply to side-constrained stochastic user equilibrium [24].

\subsection{Linear Constraint Minimization for SC-P2P-CSUE Model}

In SC-P2P-CSUE model, every arc $a \in A$ has a fixed capacity $c_{a}$. Let $\lambda$ be the multiplier of Eq. (11). Then, the total arc cost can be derived by the method of [22]:

$$
\hat{f}_{a}\left(y_{a}\right)=f_{a}\left(y_{a}\right)+\lambda_{a}
$$

where, $\lambda_{a}$ is the waiting cost for $a \in A$. Here, $\lambda_{a}$ must satisfy the following conditions:

$$
\left\{\begin{array}{l}
\lambda_{a}=0, \text { if } \quad y_{a}<c_{a} \\
\lambda_{a} \geq 0, \text { if } \quad y_{a}=c_{a}
\end{array}\right\}, a \in A
$$

Thus, the travel path cost of a traveller encompasses two parts, namely, normal travel cost and waiting cost:

$$
\hat{c}_{k}^{r}(h, \lambda)=\sum_{a \in A} \delta_{a r}^{k}\left(f_{a}\left(y_{a}\right)+\lambda_{a}\right)=c_{k}^{r}(h)+\eta_{k}^{r}(h)
$$

where,

$$
\eta_{k}^{r}(h)=\sum_{a \in A} \delta_{a r}^{k} \lambda_{a}
$$

Then, the conditions of our model can be summed up as: For any OD pair $k \in K$, the path flow $h_{k}^{r}$ equals the path flow of our model if and only if there exists a series of multipliers $\left\{\lambda_{a}, a \in A\right\}$ that satisfy:

$$
\left\{\begin{array}{l}
h_{k}^{r}=q_{k} P_{k}^{r}\left(c_{k}^{r}(h)+\eta_{k}^{r}(h)\right) \\
y_{a} \leq c_{a}, \forall a \in A \\
y_{a}=\sum_{k \in K} \sum_{r \in R_{k}} \delta_{a r} r_{k}^{r}, \forall a \in A \\
\lambda_{a}\left(y_{a}-c_{a}\right)=0, \forall a \in A \\
\lambda_{a} \geq 0, a \in A
\end{array}\right.
$$

The SC-P2P-CSUE model can be solved through linear constraint minimization [24]. In light of this, the linear constraint minimization of our model can be expressed as:

$\min z(h)=\sum_{k \in K} q_{k} S_{k}\left(\bar{c}_{k}(h)+\bar{d}_{k}\left(h_{k}\right)\right)-\sum_{k \in K} \sum_{r \in R_{k}} \bar{d}_{k}\left(h_{k}\right) h_{k}^{r}$

s.t.

$y_{a} \leq c_{a}$

$y_{a}=\sum_{k \in K} \sum_{r \in R_{k}} \delta_{a r} h_{k}^{r}, \forall a \in A$

$h_{k}^{r} \geq 0$

$\sum_{r \in R_{k}} h_{k}^{r}=q_{k}, k \in K$

where, $S_{k}$ is the satisfaction function of the path selection between OD pair $k$. Let $c_{k}(h)$ be the cost when the path flow between OD pair $k$ is $h$. Then, $(i) \mathrm{S}\left(c_{k}(h)\right)$ relative to $c_{k}(h)$ is concave; (ii) the partial derivative of $\mathrm{S}\left(c_{k}(h)\right)$ relative to $c_{k}(h)$ is the probability of the path $r$ being chosen:

$\frac{\partial S\left(c_{k}(h)\right)}{\partial c_{k}(h)}=P_{k}^{r}\left(c_{k}(h)\right)$ 


\section{EXISTENCE AND UNIQUENESS OF THE SOLUTION TO SC-P2P-CSUE MODEL}

Theorem 1 (Existence) Any local minimum $h^{*}$ of linear constraint minimization Eq. (22) - Eq. (26) satisfies the conditions of our model, and the optimal Lagrange multiplier for the arc capacity constraint equals the arc delayed cost in SC-P2P-CSUE model.

Proof: The Lagrange function of Eq. (22) - Eq. (26) can be constructed as:

$$
\begin{aligned}
& L(h, \lambda, \pi)=\sum_{k \in K} q_{k} \tilde{S}\left(\bar{c}_{k}(h)+\bar{d}_{k}\left(h_{k}\right)\right)- \\
& -\sum_{k \in K} \sum_{r \in R_{k}} \bar{d}_{k}^{r}\left(h_{k}\right) h_{k}^{r}+\sum_{a \in A} \lambda_{a}\left(y_{a}-c_{a}\right)+\sum_{k \in K} \pi_{k}\left(q_{k}-\sum_{r \in R_{k}} h_{k}^{r}\right)
\end{aligned}
$$

where, $\lambda_{a}$ and $\pi_{k}$ are the Lagrange multipliers of Eq. (23) and Eq. (26) respectively; $\bar{d}_{k}\left(h_{k}\right)$ is only a function of the flow between OD pair $k$. Taking the partial derivatives of $h_{i}^{l}\left(l \in R_{i}\right.$ is one path between OD pair $\left.i\right)$ For Eq. (28) and substituting Eq. (27) and Eq. (20) into the partial derivative formula, we have:

$$
\begin{aligned}
& \frac{\partial L(h, \lambda, \pi)}{\partial h_{i}^{l}}=\sum_{k \in K} q_{k}\left(\sum_{r \in R_{k}} P_{k}^{r}\left(\frac{\partial \bar{c}_{k}^{r}(h)}{\partial h_{i}^{l}}\right)\right)-q_{i} \sum_{r \in R_{i}}\left(P_{i}^{r}\left(\frac{\partial \bar{d}_{i}^{r}\left(h_{i}\right)}{\partial h_{i}^{l}}\right)\right) \\
& -\bar{d}_{i}^{l}\left(h_{i}\right) \sum_{r \in R_{i}}\left(\left(\frac{\partial \bar{d}_{i}^{r}\left(h_{i}\right)}{\partial h_{i}^{l}}\right) \times f_{i}^{r}\right)-\sum_{a \in A} \delta_{a r}^{k} \lambda_{a}-\pi_{i}
\end{aligned}
$$

where, $P_{k}^{r}$ and $P_{i}^{r}$ are short for the path selection probabilities $P_{k}^{r}\left(\bar{c}_{k}(h)+\bar{d}_{k}\left(h_{k}\right)\right)$ and $P_{i}^{r}\left(\bar{c}_{i}(h)+\bar{d}_{i}\left(h_{i}\right)\right)$, respectively. Then, we have:

$$
\begin{aligned}
& \sum_{k \in K} q_{k}\left(\sum_{r \in R_{k}} P_{k}^{r}\left(\frac{\partial \bar{c}_{k}^{r}(h)}{\partial h_{i}^{l}}\right)\right)=\sum_{k \in K} q_{k} \sum_{r \in R_{k}} P_{k}^{r} \sum_{a \in A}\left(\frac{\mathrm{d} \bar{f}_{a}\left(y_{a}\right)}{\mathrm{d} y_{a}}\right) \delta_{a r}^{k} \delta_{a l}^{i} \\
& =\sum_{a \in A} y_{a}\left(\frac{\mathrm{d} \bar{f}_{a}\left(y_{a}\right)}{\mathrm{d} y_{a}}\right) \delta_{a l}^{i}
\end{aligned}
$$

Thus, the Lagrange partial derivatives in Eq. (29) can be rewritten as:

$$
\frac{\partial L(h, \lambda, \pi)}{\partial h_{i}^{l}}=\sum_{a \in A} y_{a}\left(\frac{\mathrm{d} \bar{f}_{a}\left(y_{a}\right)}{\mathrm{d} y_{a}}\right) \delta_{a l}^{i}+\bar{d}_{i}^{l}\left(h_{i}\right)+\sum_{a \in A} \delta_{a l}^{i} \lambda_{a}-\pi_{i}
$$

Let $h^{*}$ be a local minimum. According to the KarushKuhn-Tucker (KKT) conditions, there exist Lagrange multipliers $\lambda^{*}$ and $\pi^{*}$ that satisfy:

$$
\begin{aligned}
& \frac{\partial L\left(h^{*}, \lambda^{*}, \pi^{*}\right)}{\partial h_{i}^{l}}=0, l \in R_{i}, i \in K \\
& y_{a}^{*} \leq c_{a} \\
& \lambda_{a}^{*}\left(y_{a}^{*}-c_{a}\right)=0 \\
& \lambda_{a}^{*} \geq 0
\end{aligned}
$$

In addition, the optimal arc flow can be derived from the path flow $h^{*}$ as:

$$
y_{a}^{*}=\sum_{k \in K} \sum_{r \in R_{k}} h_{k}^{r *} \delta_{a r}^{k}, a \in A
$$

The following can be deduced from Eq. (25):

$\bar{d}_{i}^{l}\left(h_{i}^{*}\right)=\sum_{a \in A} y_{a}^{*}\left(\frac{\overline{d f_{a}}\left(y_{a}\right)}{d y_{a}} \mid y_{a}=y_{a}^{*}\right) \delta_{a l}^{i}+\sum_{a \in A} \delta_{a l}^{i} \lambda_{a}^{*}-\pi_{i}^{*}$

$f_{i}^{l *}=q_{i} P_{i}^{l}\left(c_{i}\left(h^{*}\right)+\eta_{i}^{l *}-\pi_{i}^{*} e_{i}\right)$

where, $e_{i}=(1, \ldots, 1) ; \eta_{i}^{l *}=\left(\ldots, \eta_{i}^{l *}, \ldots\right) ; \eta_{i}^{l *}=\sum_{a \in A} \delta_{a l}^{i} \lambda_{a}^{*}$.

Obviously, the left side of Eq. (38) is the path cost under the path flow of $h^{*}$. According to the path selection probability, Eq. (38) can be transformed into:

$f_{i}^{l^{*}}=q_{i} P_{i}^{l}\left(c_{i}\left(h^{*}\right)+d_{i}^{*}\left(h^{*}\right)\right)$

Clearly, Eq. (39) is the conditions of our model. In other words, Eq. (33) - Eq. (35) and Eq. (38) satisfy the conditions of our model and $\lambda_{a}^{*}$ is the arc delayed cost.

Theorem 2 (Uniqueness) The linear constraint minimization of our model outputs a unique solution

Proof: Let $y=\left(\ldots, y_{a}, \ldots\right)$ be a row vector with all arc flows, $f_{a}\left(y_{a}\right)=\left(\ldots, f_{a}\left(y_{a}\right), \ldots\right)$ be the arc travel cost, $\Delta_{k}=\left(\delta_{a r}^{k}\right)_{|N| \times\left|R_{k}\right|}$ be the relationship between path and arc, $|N|$ be the number of arc, and $\left|R_{k}\right|$ be the number of paths in OD pair $k$. Then, the relationship between path and arc can be expressed as:

$y=\sum_{k} f_{k}\left(\Delta_{k}\right)^{\mathrm{T}}$

Considering the additivity of arc cost, the conditions of our model can be expressed as:

$y_{a}=\sum_{k \in K} q_{k} P_{k}^{a}(f(y)+\lambda)$

$y_{a} \leq c_{a}$

$\lambda_{a}\left(y_{a}-c_{a}\right)=0$

$\lambda_{a} \geq 0$

where, $P_{k}^{a}\left(f(y)-\eta\left(\lambda^{ \pm}\right)\right)$is the probability of all paths passing through arc $a$ in OD pair $k$ :

$P_{k}^{a}(f(y)+\lambda)=\sum_{k \in K} \sum_{r \in R_{k}} \delta_{a r}^{k} P_{k}^{r}\left(c_{k}(f(y))+\eta_{k}\right)$ 
Let $\boldsymbol{P}_{k}\left(c_{k}+\eta_{k}\right)$ and $\boldsymbol{P}_{k}(f(y)+\lambda)$ be the row vectors of the probabilities to select all the paths and all the arcs associated with OD pair $k$, respectively. Then, the two row vectors can be described as:

$$
\begin{aligned}
& \boldsymbol{P}_{k}\left(c_{k}+\eta_{k}\right)=\left(\ldots, P_{k}^{r}\left(c_{k}(f(y))+\eta_{k}\right), \ldots\right) \\
& \boldsymbol{P}_{k}(f(y)+\lambda)=\left(\ldots, P_{k}(f(y)+\lambda), \ldots\right)
\end{aligned}
$$

According to Eq. (45), the relationship between arc and path selection probabilities can be depicted as:

$$
\boldsymbol{P}_{k}(f(y)+\lambda)=P_{k}\left(c_{k}(f(y))+\eta_{k}\right) \Delta_{k}^{\mathrm{T}}
$$

Then, we have:

$y_{a}=\sum_{k \in K} q_{k} P_{k}(f(y)+\lambda)$

Next, Theorem 2 is proved by the reduction to absurdity.

Suppose the linearly constrained minimization Eq. (22) - Eq. (26) can generate two local minimums, namely, $f^{1}=\left(\ldots, f_{k}^{1}, \ldots\right)$ and $f^{2}=\left(\ldots, f_{k}^{2}, \ldots\right)$, with the relevant optimal Lagrange multipliers $\lambda^{1}$ and $\lambda^{2}$. Let vectors $y^{1}=\left(\ldots, y_{a}^{1}, \ldots\right)$ and $y^{2}=\left(\ldots, y_{a}^{2}, \ldots\right)$ be the path flows of our model. The two path flows, coupled with the optimal Lagrange multipliers, satisfy the conditions of our model:

$$
y^{1}-y^{2}=\sum_{k \in K} q_{k}\left(P_{k}\left(f\left(y^{1}\right)+\lambda^{1}\right)-P_{k}\left(f\left(y^{2}\right)+\lambda^{2}\right)\right)
$$

Multiplying both sides of Eq. (50) by a column vector $\left(f\left(y^{1}\right)-f\left(y^{2}\right)+\lambda^{1}-\lambda^{2}\right)^{\mathrm{T}}$, we have:

$$
\begin{aligned}
& \left(y^{1}-y^{2}\right)\left(f\left(y^{1}\right)-f\left(y^{2}\right)+\lambda^{1}-\lambda^{2}\right)^{\mathrm{T}}= \\
& =\sum_{k \in K} q_{k}\left(P_{k}\left(f\left(y^{1}\right)+\lambda^{1}\right)-P_{k}\left(f\left(y^{2}\right)+\lambda^{2}\right)\right) \times \\
& \times\left(f\left(y^{1}\right)-f\left(y^{2}\right)+\lambda^{1}-\lambda^{2}\right)^{\mathrm{T}}
\end{aligned}
$$

The first step is to prove that the above equation has a non-positive RHS. For this purpose, a real continuously differentiable function $\phi(\varsigma)=\mathfrak{R}^{1} \rightarrow \mathfrak{R}^{1}$ can be defined as:

$$
\begin{aligned}
& \phi(\varsigma)=\sum_{k \in K} q_{k}\left(P_{k}\left(f\left(y^{1}\right)+\lambda^{1}\right)-P_{k}\left(f\left(y^{2}\right)+\lambda^{2}\right)\right) \times \\
& \times\left(f\left(y^{1}\right)-f\left(y^{2}\right)+\lambda^{1}-\lambda^{2}\right)^{\mathrm{T}}
\end{aligned}
$$

It is easy to get that:

$$
\begin{aligned}
& \sum_{k \in K} q_{k}\left(P_{k}\left(f\left(y_{1}\right)+\lambda^{1}\right)-P_{k}\left(f\left(y_{2}\right)+\lambda^{2}\right)\right) \times \\
& \times\left(f\left(y_{1}\right)-f\left(y_{2}\right)+\lambda^{1}-\lambda^{2}\right)^{\mathrm{T}}=\phi(1)-\phi(0)
\end{aligned}
$$

According to the mean value theorem, there is a $\varsigma^{*} \in(1,0)$ such that:

$\phi(1)-\phi(0)=\phi^{\prime}\left(\varsigma^{*}\right)(1-0)$

The derivative of function $\phi(\varsigma)$ at $\varsigma=\varsigma^{*}$ can be expressed as:

$$
\begin{aligned}
& \phi^{\prime}\left(\varsigma^{*}\right)=\sum_{k \in K} q_{k}\left(P_{k}\left(f\left(y^{1}\right)+\lambda^{1}\right)-P_{k}\left(f\left(y^{2}\right)+\lambda^{2}\right)\right) \times \\
& \times \nabla P_{k}(\hat{f}+\hat{\lambda})\left(f\left(y^{1}\right)-f\left(y^{2}\right)+\lambda^{1}-\lambda^{2}\right)^{\mathrm{T}}
\end{aligned}
$$

where, $\nabla P_{k}(\hat{f}+\hat{\lambda})$ is the Jacobian matrix of the arc selection probabilities,

$$
\begin{aligned}
& \hat{f}=\varsigma^{*} f\left(y^{1}\right)+\left(1-\varsigma^{*}\right) f\left(y^{2}\right) \\
& \hat{\lambda}=\varsigma^{*} \lambda^{1}+\left(1-\varsigma^{*}\right) \lambda^{2}
\end{aligned}
$$

Eq. (48) implies that

$$
\nabla P_{k}(\hat{f}+\hat{\lambda})=\Delta_{k} \times \nabla P_{k}\left(\hat{c}_{k}+\hat{\eta}_{k}\right) \times \Delta_{k}^{\mathrm{T}}
$$

where, $\nabla P_{k}\left(\hat{c}_{k}+\hat{\eta}_{k}\right)$ is the Jacobian matrix of the path selection probabilities $\boldsymbol{P}_{k}(f(y)+\lambda)$. The total path travel cost $\hat{c}_{k}+\hat{\eta}_{k}$ can be defined as:

$\hat{c}_{k}=\hat{f} \Delta_{k}$

$\hat{\eta}=\hat{\lambda} \Delta_{k}$

Substituting Eq. (56) into Eq. (53), we have:

$\phi^{\prime}\left(\varsigma^{*}\right)=\sum_{k \in K} q_{k}\left(\left(f\left(y^{1}\right)-f\left(y^{2}\right)+\lambda^{1}-\lambda^{2}\right) \Delta_{k} \times\right.$
$\times \nabla P_{k}\left(\hat{c}_{k}+\hat{\eta}_{k}\right) \nabla_{k}^{T}\left(f\left(y^{1}\right)-f\left(y^{2}\right)+\lambda^{1}-\lambda^{2}\right)^{\mathrm{T}}$

Sheffi [25] pointed out that the Jacobian matrix $\nabla P_{k}\left(\hat{c}_{k}+\hat{\eta}_{k}\right)$ of the path selection probability is negative semidefinite. Thus, we have

$\phi^{\prime}\left(\varsigma^{*}\right) \leq 0$

In other words, the right side of Eq. (51) is nonpositive. The next step is to prove that left side of Eq. (51) is nonnegative. To this end, another real continuously differentiable function can be defined as:

$$
\varphi(\gamma)=f\left(y^{2}+\gamma\left(y^{1}-y^{2}\right)\right)\left(y^{1}-y^{2}\right)^{\mathrm{T}}
$$


This function has the following features:

(1) $\left(f\left(y^{1}\right)-f\left(y^{2}\right)\right)^{\mathrm{T}}\left(y^{1}-y^{2}\right)=\varphi(1)-\varphi(0)$;

(2) There exists a $\gamma^{*} \in(0,1) \quad$ satisfying $\left(f\left(y^{1}\right)-f\left(y^{2}\right)\right)^{\mathrm{T}}\left(y^{1}-y^{2}\right)=\varphi^{\prime}\left(\gamma^{*}\right)(1-0)$;

(3) $\varphi^{\prime}\left(\gamma^{*}\right)=\left(y^{1}-y^{2}\right) \nabla f\left(y^{2}+\gamma^{*}\left(y^{1}-y^{2}\right)\right)\left(y^{1}-y^{2}\right)^{\mathrm{T}}$,

where, $\nabla f\left(y^{2}+\gamma^{*}\left(y^{1}-y^{2}\right)\right)$ is the Jacobian matrix of $f(y)$ when $y=y^{2}+\gamma^{*}\left(y^{1}-y^{2}\right)$.

Since $\nabla f\left(y^{2}+\gamma^{*}\left(y^{1}-y^{2}\right)\right)$ is a diagonal matrix of non-negative real numbers $f_{a}\left(y^{2}+\gamma^{*}\left(y^{1}-y^{2}\right)\right)$, we have:

$\varphi^{\prime}\left(\gamma^{*}\right) \geq 0$

Feature (2) implies that:

$\left(f\left(y^{1}\right)-f\left(y^{2}\right)\right)^{\mathrm{T}}\left(y^{1}-y^{2}\right) \geq 0$

Further, we have:

$$
\begin{aligned}
& \left(y^{1}-y^{2}\right)\left(\lambda^{1}-\lambda^{2}\right)^{\mathrm{T}}=\sum_{a \in A}\left(y_{a}^{1}-y_{a}^{2}\right)\left(\lambda_{a}^{1}-\lambda_{a}^{2}\right)= \\
& =\sum_{a \in A}\left(\lambda_{a}^{1}\left(c_{a}-y_{a}^{1}\right)+\lambda_{a}^{2}\left(c_{a}-y_{a}^{2}\right)\right) \geq 0
\end{aligned}
$$

Because $\lambda_{a} \geq 0$ and $c_{a}-y_{a} \geq 0$, it is easy to derive that $\left(y^{1}-y^{2}\right)\left(\lambda^{1}-\lambda^{2}\right)^{\mathrm{T}} \geq 0$.

Then, the left side of Eq. $\left(y^{1}-y^{2}\right)\left(f\left(y^{1}\right)-f\left(y^{2}\right)+\lambda^{1}-\lambda^{2}\right)^{\mathrm{T}}$ can be divided into two parts: $\quad\left(f\left(y^{1}\right)-f\left(y^{2}\right)\right)^{\mathrm{T}}\left(y^{1}-y^{2}\right) \quad$ and $\left(y^{1}-y^{2}\right)\left(\lambda^{1}-\lambda^{2}\right)^{\mathrm{T}}$, as shown in Eq. (63) and Eq. (64).

Since $\quad\left(f\left(y^{1}\right)-f\left(y^{2}\right)\right)^{\mathrm{T}}\left(y^{1}-y^{2}\right) \geq 0 \quad$ and $\left(y^{1}-y^{2}\right)\left(\lambda^{1}-\lambda^{2}\right)^{\mathrm{T}} \geq 0 \quad, \quad$ we $\quad$ have $\left(y^{1}-y^{2}\right)\left(f\left(y^{1}\right)-f\left(y^{2}\right)+\lambda^{1}-\lambda^{2}\right)^{\mathrm{T}} \geq 0$.

The above analysis shows that the left side of Eq. (51) is greater than or equal to 0 , while the right side of Eq. (51) is smaller than or equal to 0 , that is, both sides of the equation could be equal to 0 :

$\left(f\left(y^{1}\right)-f\left(y^{2}\right)\right)^{\mathrm{T}}\left(y^{1}-y^{2}\right)=0$
Since the arc travel cost $f(y)$ is a strictly monotone increasing function associated with $y$, we have $y^{1}-y^{2}=0$. Therefore, we have.

In conclusion, $y^{1}=y^{2}$, that is, the linear constraint minimization of our model outputs a unique solution.

\section{COMPUTATION RESULTS}

In this section, we initially propose a solution to solve the linear constrained minimization problem of SC-P2PCSUE model. Then, we examine our proposed model on a four-node transportation network to demonstrate the solution optimality of our developed algorithm.

\subsection{Linear Constraint Minimization Solutions of SC-P2P. CSUE Model}

The linear constraint minimization cannot be implemented directly due to the lack of specific expression of the path delay $d(h)$ in the objective function Eq. (22). This paper settles this problem with Lagrange duality [26]:

$\max _{\lambda \geq 0} L(\lambda)$

where, $\lambda=\left(\ldots, \lambda_{a}, \ldots\right)$ is a vector of the Lagrange multipliers associated with constraint Eq. (22). Then,

$$
L(\lambda)=\min _{h \in \Omega}\left(z(h)+\sum_{a \in A} \lambda_{a}\left(y_{a}-c_{a}\right)\right)
$$

where, $\Omega$ is the set of all feasible path flows without considering arc capacity $\Omega=(h \mid h)$ satisfies Eq. (22) - Eq. (26). Eq. (66) shows that Lagrange duality is the optimal solution of linear constraint minimization associated with $\lambda$. Note that $L(\lambda)$ is a concave function associated with nonnegative $\lambda$.

Theorem 3 SC-P2P-CSUE model is a no constraint maximization problem. The objective function is a continuous differentiable concave function. If $\lambda^{*}$ is the optimal Lagrange duality, then $y\left(\lambda^{*}\right)$ must be the arc flow of SC-P2P-CSUE model.

Proof: For a given $\lambda \geq 0$, suppose $y(\lambda)=\left(\ldots, y_{a}(\lambda), \ldots\right)$ is a local minimum of linear constrained minimization. According to Theorem 2, Theorem 6.3.3 of Bazaraa et al. and Theorem 6.3.1 of Bazaraa et al., it is known that $y(\lambda)$ is unique, and $L(\lambda)$ is continuous differentiable and concave [20].

The KKT conditions of Lagrange duality are as follows:

$$
\begin{aligned}
& \frac{\partial L\left(h^{*}, \lambda^{*}, \pi^{*}\right)}{\partial h_{i}^{l}}=0 \\
& \frac{\partial L\left(h^{*}, \lambda^{*}, \pi^{*}\right)}{\partial \pi_{k}}=0 \\
& y_{a}^{*} \leq c_{a}
\end{aligned}
$$


$\lambda_{a}^{*}\left(y_{a}^{*}-c_{a}\right)=0$

$\lambda_{a}^{*} \geq 0$

Similar to the proof process of Theorem 2, the KKT conditions must satisfy the conditions of SC-P2P-CSUE model.

\subsection{Case Study}

The target network has 4 nodes and 5 arcs. For simplicity, the author only tested the path flow of one OD pair (Fig. 2). The demand $q$ of the OD pair $(1,4)$ was set to 400. The details on $t_{a}, l_{a}$, and $c_{a}$ are shown in Tab. 3. The BRP function was adopted to describe the congestion cost, with $\alpha=0,15$ and $\beta=2$.

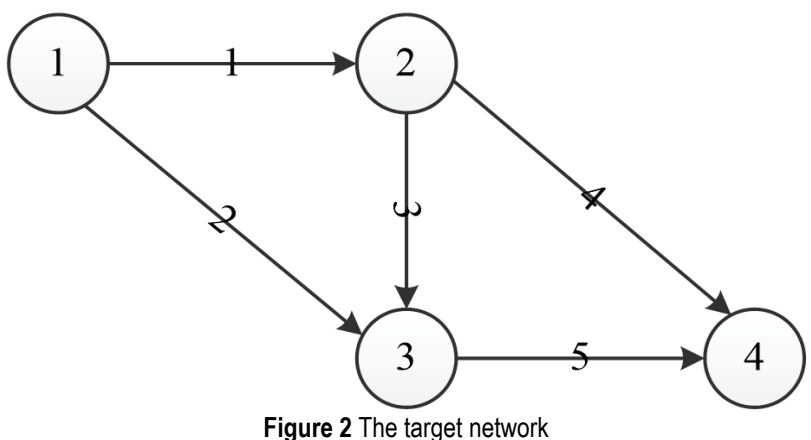

Table 3 Details of the target network

\begin{tabular}{|c|c|c|c|c|c|}
\hline \multirow{2}{*}{ Parameters } & \multicolumn{5}{|c|}{ No. of $\operatorname{arc} a$} \\
\cline { 2 - 6 } & 1 & 2 & 3 & 4 & 5 \\
\hline$t_{a}$ & 6 & 5 & 3 & 6 & 3 \\
\hline$l_{a}$ & 4 & 3 & 1 & 4 & 1 \\
\hline$c_{a}$ & 300 & 200 & 250 & 350 & 400 \\
\hline
\end{tabular}

Moreover, the unit distance cost of drivers $\rho$ was set to $2 \mathrm{USD} / \mathrm{km}$, the distance-time conversion factor $\eta$ was set to $1 \mathrm{USD} / \mathrm{min}$, the value of $\gamma$ was set to 4 , and the inconvenient coefficients were set to $\mu_{a}^{d}=\mu_{a}^{p}=0,02$ and $\pi_{a}^{d}=\pi_{a}^{p}=0,0002$, respectively.

Table 4 Arc flows and costs in the network $(\rho=2)$

\begin{tabular}{|c|c|c|c|c|c|c|c|}
\hline$a$ & $y_{a}$ & $f_{a}(y)$ & $\lambda$ & $a$ & $y_{a}$ & $f_{a}(y)$ & $\lambda$ \\
\hline$a_{11}$ & 0 & 14 & 0 & $a_{33}$ & 160 & 4,81 & 0 \\
\hline$a_{12}$ & 40 & 8,82 & 0 & $a_{41}$ & 0 & 14 & 0 \\
\hline$a_{13}$ & 160 & 9,12 & 0 & $a_{42}$ & 0 & 14 & 0 \\
\hline$a_{21}$ & 0 & 11 & 0,85 & $a_{43}$ & 0 & 14 & 0 \\
\hline$a_{22}$ & 40 & 7,35 & 0,95 & $a_{51}$ & 0 & 5 & 0,73 \\
\hline$a_{23}$ & 160 & 8,07 & 0,47 & $a_{52}$ & 80 & 5,66 & 1,48 \\
\hline$a_{31}$ & 0 & 5 & 0 & $a_{53}$ & 320 & 6,11 & 0,36 \\
\hline$a_{32}$ & 40 & 4,52 & 0 & & & & \\
\hline
\end{tabular}

It can be seen from Tab. 4 that the divers and riders prefer carpooling in the network. More notably, the number of riders passing arc 2 reached the capacity constraint of that arc. According to the equilibrium conditions in Eq. (18), the value of $\lambda$ must be greater than 0 . In this case, the associated values of $\lambda$ were $\lambda_{22}=0,95$ and $\lambda_{23}=0,47$, that is, the delayed congestion costs. Arc 5 also reached its capacity constraint, where the associated values of $\lambda$ were $\lambda_{52}=1,48$ and $\lambda_{53}=0,36$.

The path costs of the network under fixed parameter values are recorded in Tab. 5 .
Table 5 Path costs in the network

\begin{tabular}{|c|c|c|c|}
\hline Path & $1-3-4$ & $1-2-3-4$ & $1-2-4$ \\
\hline Solo driver & 17,58 & 24,73 & 28 \\
\hline Carpooling driver & 15,44 & 20,48 & 22,82 \\
\hline Carpooling rider & 15,01 & 20,4 & 23,12 \\
\hline
\end{tabular}

Tab. 5 shows that the travellers favoured the path '1-34 '. Using this path, the solo drivers had the highest travel cost $(17,58)$. Thus, no driver would like to choose solo driving. Meanwhile, the travel cost of the riders was minimized under this path, and encouraged riders to choose carpooling. Due to the arc capacity constraint, a portion of travellers had to choose '1-2-3-4'.

\subsubsection{The Effect of Fuel Price}

Next, the unit distance cost of drivers $\rho$ was adjusted to $0,5 \mathrm{USD} / \mathrm{km}$, while the other parameter values were kept the same. In this case, obvious changes took place in the arc costs of the network.

Table 6 Arc flows and costs in the network $(\rho=0,5)$

\begin{tabular}{|c|c|c|c|c|c|c|c|}
\hline$a$ & $y_{a}$ & $f_{a}(y)$ & $\lambda$ & $a$ & $y_{a}$ & $f_{a}(y)$ & $\lambda$ \\
\hline$a_{11}$ & 100 & 8,1 & 0 & $a_{33}$ & 80 & 3,95 & 0 \\
\hline$a_{12}$ & 20 & 7,16 & 0 & $a_{41}$ & 0 & 8 & 0 \\
\hline$a_{13}$ & 80 & 7,36 & 0 & $a_{42}$ & 0 & 8 & 0 \\
\hline$a_{21}$ & 102 & 6,78 & 0,45 & $a_{43}$ & 0 & 8 & 0 \\
\hline$a_{22}$ & 19,6 & 6,42 & 0,38 & $a_{51}$ & 202 & 3,57 & 0,33 \\
\hline$a_{23}$ & 78,4 & 6,61 & 0,42 & $a_{52}$ & 39,6 & 4,37 & 0,32 \\
\hline$a_{31}$ & 100 & 3,27 & 0 & $a_{53}$ & 158,4 & 4,66 & 0,25 \\
\hline$a_{32}$ & 20 & 3,77 & 0 & & & & \\
\hline
\end{tabular}

As shown in Tab. 6, the proportion of carpooling changed greatly as the unit distance cost of drivers dropped to $0,5 \mathrm{USD} / \mathrm{km}$. Under $\rho=0,5$, the lowest cost path in the network was still '1-3-4'. At this moment, the solo drivers had the lowest cost, such that many carpooling drivers turned to solo driving. This means the decline of fuel price encourages the travelers to drive alone, and the inverse is also true. The results show that fuel price adjustment can effectively stimulate the enthusiasm in carpooling and thus ease the traffic congestion.

\subsubsection{The Effect of Inconvenient Cost}

Keeping the other parameter values unchanged, the inconvenient coefficients were adjusted to $\mu_{a}^{d}=\mu_{a}^{p}=1$ and $\pi_{a}^{d}=\pi_{a}^{p}=1$, respectively. It is easy to learn that path '1-3-4' boasted the lowest cost. At this moment, the solo drivers had the lowest cost $(11,2)$, such that all carpooling participants were willing to select solo-driving. In practice, the carpooling is made inconvenient by the following factors: the time and distance of detouring, the waiting time, the reduced comfort, and the commission of the carpooling platform. When these factors push up the cost to an excessively high level, the travellers will leave the platform and choose to travel alone.

\section{CONCLUSIONS}

In this paper, a super-network for P2P carpooling is constructed, involving the solo drivers, carpooling drivers and carpooling riders. Then, the cost functions were formulated for each of the three types of travellers. Based on the super-network, the authors established a side- 
constrained P2P carpooling stochastic user equilibrium model and analyzed the details about the conditions of our model. Next, the linear constrain minimization of our model was proved to have a unique solution, laying the basis for $\mathrm{P} 2 \mathrm{P}$ carpooling and traffic assignment. This research mainly focuses on model construction, failing to validate the model through in-depth case studies, and only considers two travel modes: solo driving and carpooling.

We hope that after SC-P2P-CSUE model applied to public transportation, more private cars and drivers are introduced, which potentially benefits traffic. The future research will introduce more travel modes to the model, making it more realistic and accurate. Then, we also will try our best to gather more actual data and look for cooperative enterprises for application creation.

\section{Acknowledgement}

This work was supported by Humanities and social sciences fund of the Ministry of Education (No.19YJC630119) and National Natural Science Foundation of China (NSFC) (No. 71871206).

\section{REFERENCES}

[1] Schrank, D., Eisele, B., \& Lomax, T. (2012). TTI's 2012 urban mobility report. The Texas A\&M University System.

[2] Masoud, N. \& Jayakrishnan, R. (2017). A real-time algorithm to solve the peer-to-peer ride-matching problem in a flexible ridesharing system. Transportation Research Part B: Methodological, 106, 218-236. https://doi.org/10.1016/j.trb.2017.10.006

[3] Ma, R., Yao, L., Song, L., \& Jin, M. (2019). A novel algorithm for peer-to-peer ridesharing match problem. Neural Computing and Applications, 31(1), 247-258. https://doi.org/10.1007/s00521-018-3733-5

[4] Bimpikis, K., Candogan, O., \& Saban, D. (2019). Spatial pricing in ride-sharing networks. Operations Research, 67(3), 599-604. https://doi.org/10.1287/opre.2018.1800

[5] Lu, W. \& Quadrifoglio, L. (2019). Fair cost allocation for ridesharing services -modeling, mathematical programming and an algorithm to find the nucleolus. Transportation Research Part B: Methodological, 121, 41-55. https://doi.org/10.1016/j.trb.2019.01.001

[6] Sun, H., Wang, H., \& Wan, Z. (2019).Model and analysis of labor supply for ride-sharing platforms in the presence of sample self-selection and endogeneity. Transportation Research Part B: Methodological, 125, 76-93. https://doi.org/10.1016/j.trb.2019.04.004

[7] Salamanis, A., Kehagias, D. D., Tsoukalas, D., \& Tzovaras, D. (2019). Reputation assessment mechanism for ridesharing applications based on clustering user travel preferences. International Journal of Transportation Science and Technology, 8(1), 68-81. https://doi.org/10.1016/j.ijtst.2018.08.002

[8] Sánche, D., Martínez, S. \& Domingo, F. J. (2016). Co-utile P2P ridesharing via decentralization and reputation management. Transportation Research Part C: Emerging Technologies, 73, 147-166. https://doi.org/10.1016/j.trc.2016.10.017

[9] Burtch, G., Carnahan, S., \& Greenwood, B. N. (2018). Can you gig it? An empirical examination of the gig economy and entrepreneurial activity. Management Science, 64(12), 54975520. https://doi.org/10.1287/mnsc.2017.2916

[10] Yu, B., Ma, Y., Xue, M., Tang, B., Wang, B., Yan, J., \&Wei, Y. (2017). Environmental benefits from ridesharing: A case of Beijing. Applied Energy, 191, 141-152. https://doi.org/10.1016/j.apenergy.2017.01.052

[11] Yin, B., Liu, L., Coulombel, N., \& Viguie, V. (2018).Appraising the environmental benefits of ridesharing: The Paris region case study. Journal of cleaner production, 177, 888-898. https://doi.org/10.1016/j.jclepro.2017.12.186

[12] Alexander, L. P. \& González, M. C. (2016). Assessing the Impact of Real-time Rides haring on Urban Traffic using Mobile Phone Data, in Proc. UrbComp'15, Sydney, Australia, 1-9.

[13] Rayle, L., Dai, D., Chan, N., Cervero, R., \& Shaheen, S. (2016) Just a better taxi? A survey-based comparison of taxis, transit, and ridesourcing services in San Francisco. Transport Policy, 45, 168-178. https://doi.org/10.1016/j.tranpol.2015.10.004

[14] Li, Z., Hong, Y., \& Zhang, Z. (2016). Do ride-sharing services affect traffic congestion? An empirical study of uber entry. Social Science Research Network, 2002, 1-29. https://doi.org/10.2139/ssrn.2838043

[15] Li, Z., Hong, Y., \& Zhang, Z. (2017). An empirical analysis of on-demand ride sharing and traffic congestion. Proc. HICSS, Hilton Waikoloa Village, USA, 4-13. https://doi.org/10.24251/HICSS.2017.002

[16] Erhardt, G. D., Roy, S., Cooper, D., Sana, B., Chen, M., \& Castiglione, J. (2019).Do transportation network companies decrease or increase congestion? Science Advances, 5(5), eaau2670. https://doi.org/10.1126/sciadv.aau2670

[17] Yang, H. \& Huang, H. J. (1999). Carpooling and congestion pricing in a multilane highway with high-occupancy-vehicle lanes. Transportation Research Part A: Policy and Practice, 33(2), 139-155. https://doi.org/10.1016/S0965-8564(98)00035-4

[18] Qian, Z. S. \& Zhang, H. M. (2011). Modeling multi-modal morning commute in a one-to-one corridor network. Transportation Research Part C: Emerging Technologies, 19(2), 254-269. https://doi.org/10.1016/j.trc.2010.05.012

[19] Xu, H., Ordóñez, F., \& Dessouky, M. (2014). A traffic assignment model for a ridesharing transportation market. Journal of Advanced Transportation, 49(7), 793-816. https://doi.org/10.1002/atr.1300

[20] Xu, H., Pang, J. S., Ordóñez, F., \& Dessouky, M. (2015). Complementarity models for traffic equilibrium with ridesharing. Transportation Research Part B: Methodological, 81, 161-182. https://doi.org/10.1016/j.trb.2015.08.013

[21] Dafermos, S. C. \& Sparrow, F. T. (1969). The traffic assignment problem for a general network. Journal of Research of the National Bureau of Standards B, 73(2), 91118. https://doi.org/10.6028/jres.073B.010

[22] Larsson, T. \& Patriksson, M. (1999). Side constrained traffic equilibrium models - analysis, computation and applications. Transportation Research Part B: Methodological, 33(4), 233-264. https://doi.org/10.1016/S0191-2615(98)00024-1

[23] Patriksson, M. \& Larsson, T. (1994). Equilibrium characterizations of solutions to side constrained asymmetric traffic assignment models. Le Matematiche, 49(2), 249-280.

[24] Meng, Q., Lam, W. H. K., \& Yang, L. (2008). General stochastic user equilibrium traffic assignment problem with link capacity constraints. Journal of Advanced Transportation, 42(4), 429-466. https://doi.org/10.1002/atr.5670420403

[25] Sheffi, Y. (1985). Urban transportation networks: equilibrium analysis with mathematical programming methods. Transportation Science, 19(4), 463-466.

[26] Bazaraa, M. S., Sherali, H. D., \& Shetty, C. M. (1993). Nonlinear programming: theory and algorithms. New York, USA: John Wiley \& Sons 


\section{Contact information:}

Ruimin MA, Lecturer

School of Management, Guangzhou University,

Guangzhou 510006, China

E-mail:1486857526@qq.com

\section{Lifei YAO, Lecturer}

(Corresponding author)

School of geography and tourism,

Guangdong University of Finance \& Economics,

Guangzhou 510320, China

E-mail: yaolf1218@gdufe.edu.cn 